\title{
Hepatic Uptake of Bile Acids in Man
}

\author{
FASTING AND POSTPRANDIAL CONCENTRATIONS OF INDIVIDUAL BILE \\ ACIDS IN PORTAL VENOUS AND SYSTEMIC BLOOD SERUM
}

\author{
Bo ANgelin, Ingemar Björkhem, Kurt Einarsson, and Staffan Ewerth, \\ Departments of Medicine, Clinical Chemistry, and Surgery, Karolinska \\ Institutet at Huddinge University Hospital, Stockholm, Sweden
}

A B S T R A C T This investigation was undertaken in order to $(a)$ characterize the postprandial inflow of individual bile acids to the liver and $(b)$ determine if peripheral venous bile acid levels always adequately reflect the portal venous concentration, or if saturation of hepatic bile acid uptake can occur under physiological conditions. In five patients with uncomplicated cholesterol gallstone disease, the umbilical cord was cannulated during cholecystectomy, and a catheter was left in the left portal branch for 5 to $7 \mathrm{~d}$. The serum concentrations of cholic acid, chenodeoxycholic acid, and deoxycholic acid in portal venous and systemic circulation were then determined at intervals of 15 to $30 \mathrm{~min}$ before and after a standardized meal. A highly accurate and specific gas chromatographic/ mass spectrometric technique was used.

The sum of the fasting concentrations of the three bile acids averaged $14.04 \pm 4.13 \mu \mathrm{mol} / \mathrm{liter}$ in portal venous serum, and $2.44 \pm 0.31 \mu \mathrm{mol} /$ liter in peripheral venous serum. The estimated hepatic fractional uptake of cholic acid was $\sim 90 \%$, and those of chenodeoxycholic acid and deoxycholic acid were $70-80 \%$. This resulted in an enrichment of systemic bile acids in the dihydroxy bile acid species. In response to a standardized meal, portal venous bile acid concentrations increased two- to sixfold, with a peak seen $15-60 \mathrm{~min}$ after the meal. The maximum postprandial portal venous bile acid concentration averaged $43.04 \pm 6.12$ $\mu \mathrm{mol} /$ liter, and the corresponding concentration in peripheral serum was $5.22 \pm 0.74 \mu \mathrm{mol} /$ liter. The estimated fractional uptakes of the individual bile acids were not affected by the increased inflow to the liver. The peripheral venous concentrations of individual as

Received for publication 12 March 1982 and in revised form 9 June 1982.

Dr. Angelin is the recipient of a research fellowship from the Ernst Klenk Foundation. well as total bile acids were well correlated with those in portal venous serum.

The results $(a)$ give a quantitation of postprandial bile acid inflow to the liver and $(b)$ indicate that the hepatic uptake system for bile acids in healthy man cannot be saturated during maximal inflow of endogenous bile acids. Measurement of peripheral serum bile acids can thus give important information on the status of the enterohepatic circulation.

\section{INTRODUCTION}

In man, the two primary bile acids, cholic acid $(\mathrm{C})^{1}$ and chenodeoxycholic acid (CD), together with the secondary bile acid, deoxycholic acid (D), undergo an efficient enterohepatic circulation (1). Secreted as glycine or taurine conjugates by the liver, the bile acids are reabsorbed in the small intestine and returned to the liver in the portal vein. The portal venous inflow of bile acids is of regulatory importance for the hepatic bile secretion, and for the conversion of cholesterol to bile acids (1-4). Due to the efficient hepatic uptake, fasting serum bile acid concentrations are generally low in peripheral circulation, in spite of a significant inflow of bile acids to the liver in the portal vein (5). In response to a meal, peripheral venous levels of serum bile acids increase severalfold $(6,7)$. This is presumed to be the consequence of an increased absorption of bile acids, and thus an elevated portal venous load to the liver.

By direct measurement of the individual bile acids in portal venous and systemic circulation we have shown that there are differences in intestinal uptake and hepatic clearance of individual bile acids in fasting man (5). The relation between individual bile acid concentrations in portal venous and systemic circula-

\footnotetext{
${ }^{1}$ Abbreviations used in this paper: $\mathrm{C}$, cholic acid; CD, chenodeoxycholic acid; D, deoxycholic acid.
} 
tion during a meal has not been studied previously in man. Thus it is not known if peripheral venous bile acid levels always adequately reflect the portal venous concentration, or if saturation of hepatic bile acid uptake can occur under physiological conditions. To address these questions, we have determined the concentrations of $\mathrm{C}, \mathrm{CD}$, and $\mathrm{D}$, using an accurate gas chromatographic/mass spectrometric technique, in portal venous and systemic blood serum of cholecystectomized subjects fed a standardized meal.

\section{METHODS}

Patients. Five patients, one male and four females, were included in the study. They were all referred to the hospital because of uncomplicated gallstone disease. The stones consisted of $>70 \%$ cholesterol in all cases. None of the patients had a history of common duct stones, and they all had a normal operative cholangiogram. Histological examination of operative liver biopsies was normal in all cases. Basal data on the patients are presented in Table I. None of them had clinical or laboratory evidence of diabetes mellitus, hyperlipidemia, or diseases affecting kidney or thyroid function. Except for a slightly elevated serum bilirubin concentration in one of the patients, they all displayed normal liver function tests. None of the patients were on treatment with drugs.

Experimental procedure. All patients had an uneventful cholecystectomy under standardized conditions as described $(5,8)$. During laparotomy, the umbilical vein was identified and cannulated as described (9). The catheter was inserted into the left portal branch, close to the main stem, and filled with a heparin solution. There was no evidence of complications in any of the patients, and they all had a normal postoperative recovery and mobilization. A detailed description of the surgical procedure, together with a discussion of the prevention of possible complications, has been published recently $(9)$.

After 5-7 d, when the patients had been given oral meals for 3-4 d, and their bowel habits were normal, they were studied in the morning after a 12-h fast. At 7:30 a.m., an indwelling needle was inserted into an antecubital vein, and two to three blood samples were drawn from peripheral and portal venous circulation in the fasting state. At 8:00 a.m., the patients were given a standardized breakfast, consisting of $2 \mathrm{dl}$ milk, two cheese sandwiches, and $2 \mathrm{dl}$ coffee. The energy content of this meal is equivalent to $1,750 \mathrm{~kJ}$ with carbohydrate, fat, and protein accounting for 33,44 , and $23 \%$ of total, respectively. $5 \mathrm{ml}$ of blood were then drawn from the peripheral venous and portal venous catheters at intervals of $15 \mathrm{~min}$ for $150-240 \mathrm{~min}$. The portal venous catheter could then be withdrawn without complications, and the patients were discharged from the hospital on the following day.

All patients had given their informed consent before surgery, and the ethical aspects of the study had been approved by the Ethical Committee of Karolinska Institutet.

Analytical procedures. After clotting of blood at room temperature, serum was obtained by centrifugation and frozen at $-20^{\circ} \mathrm{C}$ for subsequent analysis. To $0.50 \mathrm{ml}$ of peripheral and $0.10 \mathrm{ml}$ of portal venous serum, $2.5 \mu \mathrm{g}$ of $\left[2,2,3,4,4,{ }^{2} \mathrm{H}_{5}\right] \mathrm{C}, 2.5 \mu \mathrm{g}$ of $\left[2,2,3,4,4-{ }^{2} \mathrm{H}_{5}\right] \mathrm{CD}$, and $5.0 \mu \mathrm{g}$ of $\left[11,11,12-{ }^{2} \mathrm{H}_{3}\right] \mathrm{D}$, dissolved in acetone, were added. The serum, with the added internal standards, was hydrolyzed with $1 \mathrm{M} \mathrm{KOH}$ at $110^{\circ} \mathrm{C}$ for $12 \mathrm{~h}$. The alkaline solution was extracted three times with diethyl ether to remove most of the neutral steroids. The bile acids were then extracted from the acidified water phase with ethyl ether, methylated with diazomethane, and converted into trimethylsilyl ether derivatives. The derivatives were analyzed by gas chromatography-mass spectrometry using an LKB 9000 instrument equipped with a multiple ion detector (MIID) unit. A $1.5 \%$ SE-30 column was used, and the operating temperature was $230^{\circ}-270^{\circ} \mathrm{C}$.

Our described mass fragmentographic technique (10) for determination of the individual serum bile acids, C, CD, and $D$, has been modified, using more specific ions for each bile acid derivative. As a consequence, however, each bile acid must be determined separately with our instrument (LKB 9000 ). Thus, $C$ was analyzed with two of the channels focused on $m / e 623$ and $m / e 628$, corresponding to the $M-15$ peak in the mass spectrum of trimethylsilyl derivative of unlabeled and deuterium-labeled $C$, respectively. $C D$ was analyzed with two of the channels focused on $\mathrm{m} / e \mathbf{3 7 0}$ and 373 , corresponding to the $M-2 \times 90$ peak in the mass spectrum of trimethylsilyl derivative of unlabeled and deuterium-labeled $C D$, respectively. $D$ was analyzed with two of the channels focused on $m / e 255$ and 258, corresponding to the base peak in the mass spectrum of trimethylsilyl deriv-

TABLE I

Basal Data on the Patients

\begin{tabular}{|c|c|c|c|c|c|c|c|}
\hline Patient no. & Sex ${ }^{*}$ & Age & RBWI & Bilirubin & ASAT\& & ALAT\$ & AP\$ \\
\hline & & & & 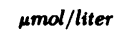 & & $\mu k a t / l i t e r$ & \\
\hline 1 & $\mathbf{M}$ & 68 & 115 & 28.0 & 0.33 & 0.41 & 2.6 \\
\hline 2 & $\mathbf{F}$ & 67 & 103 & 11.0 & 0.40 & 0.48 & 3.0 \\
\hline 3 & $\mathbf{F}$ & 64 & 117 & 4.4 & 0.50 & 0.36 & 3.6 \\
\hline 4 & $\mathbf{F}$ & 58 & 99 & 9.0 & 0.37 & 0.37 & 2.5 \\
\hline 5 & $\mathbf{F}$ & 55 & 88 & 11.0 & 0.41 & 0.42 & 4.4 \\
\hline Mean \pm SEM & & $62 \pm 3$ & $104 \pm 5$ & $11.1 \pm 2.5$ & $0.40 \pm 0.03$ & $0.41 \pm 0.02$ & $3.2 \pm 0.4$ \\
\hline
\end{tabular}

- M, male; F, female.

I Relative body weight, calculated as weight $(\mathrm{kg}) /[$ height $(\mathrm{cm})-100$ ] $\times 100 \%$.

$\$$ Normal range: bilirubin, 3.4 to 20.5 ; ASAT (aspartate aminotransferase) $<0.70$; ALAT (alanine aminotransferase) < 0.70; AP (alkaline phosphatase) $<5.4$. 
ative of unlabeled and deuterium-labeled $D$, respectively. The concentrations of the individual bile acids were thus calculated from the ratios $\mathrm{C} /\left[2,2,3,4,4-{ }^{2} \mathrm{H}_{5}\right] \mathrm{C}$ (tracing at $\mathrm{m} /$ $e$. 623/tracing at $m / e$ 628), CD/[2,2,3,4,4- $\left.{ }^{2} \mathrm{H}_{5}\right] \mathrm{CD}$ (tracing at $m / e 370 /$ tracing at $m / e 373)$, and $\mathrm{D} /\left[11,11,12-{ }^{2} \mathrm{H}_{3}\right] \mathrm{D}$ (tracing at $m / e 255 /$ tracing at $m / e$ 258). Standard curves were used for each bile acid. The relative standard deviation of the method, as calculated from duplicate samples, was $2-3 \%$.

\section{RESULTS}

The sum of the fasting concentrations of $\mathrm{C}, \mathrm{CD}$, and $D$ averaged $14.04 \pm 4.13 \mu \mathrm{mol} /$ liter (mean $\pm S E M$ ) in portal venous serum, and $2.44 \pm 0.31 \mu \mathrm{mol} / \mathrm{liter}$ in peripheral venous serum. With the exception of patient 4 , who had a relative dominance of $C$ in both portal venous and peripheral serum, the concentrations of the three bile acids were roughly similar in the portal vein (Table II). In all cases, the ratio between portal venous and peripheral venous concentration was higher for $C$ than for $C D$ or $D$, indicating a more efficient hepatic uptake of the trihydroxy bile acid. Patient 1 , who displayed a slightly elevated serum bilirubin concentration, also had the lowest ratios between portal venous and peripheral venous concentrations for all three bile acids. However, the fasting serum bile acid concentrations of this patient were not increased (Table II).

In response to the meal, there was a rapid rise in the portal venous as well as the peripheral venous concentration of all three bile acids. In all patients, there was a tendency to an earlier rise of the bile acid concentration in the portal vein than in the peripheral circulation. Two representative examples are seen in Figs. 1 and 2 (patients 2 and 4 ). A first peak concentration occurred simultaneously for all three bile acids within 15 to $60 \mathrm{~min}$ after the meal (Table III). Bile acid concentrations then decreased somewhat over a 30-60-min period, whereafter a second peak (generally lower) occurred $\sim 90 \mathrm{~min}$ after the initial one.

The sum of the concentrations of $\mathrm{C}, \mathrm{CD}$, and $\mathrm{D}$ at the maximum of the first peak averaged $43.04 \pm 6.12$ $\mu \mathrm{mol} /$ liter in portal venous and 5.22 $\pm 0.74 \mu \mathrm{mol} / \mathrm{liter}$ in peripheral venous serum, respectively (Table III). The ratio between portal venous and peripheral venous concentration was higher for $C$ than for $C D$ or $D$ also during maximal inflow of bile acids. Again, patient 1 displayed lower ratios, whereas his peripheral venous bile acid levels were not clearly distinguishable from those of the others.

The data thus indicated an efficient uptake of all three bile acids both in the fasting and in the postprandial state. To make an estimation of the fractional uptake of the individual bile acids, we have to assume that the concentration of the bile acids in hepatic venous blood serum is similar to that in the peripheral circulation. This assumption was validated by studies in five healthy volunteers, undergoing hepatic venous catheterization as part of an investigation on carbohydrate metabolism (cf. 11). As seen in Table IV, simultaneously determined hepatic venous and arterial concentrations of $C, C D$, and $D$ were in all cases except one almost identical. Furthermore, as we could not directly determine blood flow, we have to assume a constant ratio of $1: 3$ between the hepatic arterial and the portal venous flow (cf. 5). In the fasting state, this estimated uptake was $\sim 85 \%$ for $\mathrm{C}$ and lower, $\sim 70 \%$, for CD and D (Table V). Patient 1 had a clearly reduced fractional uptake of all three bile acids. The corresponding values were similar-or even slightly higher-for all three bile acids during maximal postprandial inflow. The estimated fractional uptake of $\mathrm{C}$ was again higher than that of $C D$ or $D$.

Although there was some scattering of the data points, the peripheral venous concentration of an in-

TABLE II

Fasting Concentrations of Individual Bile Acids in Portal Venous and Peripheral Venous Serum of Five Cholecystectomized Patients ${ }^{\circ}$

\begin{tabular}{|c|c|c|c|c|c|c|c|c|c|}
\hline \multirow[b]{2}{*}{ Patient no. } & \multicolumn{3}{|c|}{ Cholic acid } & \multicolumn{3}{|c|}{ Chenodeoxycholic acid } & \multicolumn{3}{|c|}{ Deoxycholic acid } \\
\hline & $P$ & $\mathrm{v}$ & $\mathrm{P} / \mathrm{V}$ & $\mathbf{P}$ & $v$ & $\mathrm{P} / \mathrm{V}$ & $\mathbf{P}$ & $\mathrm{v}$ & $\mathbf{P} / \mathrm{V}$ \\
\hline & umol/liter & $\mu \mathrm{mol} / \mathrm{liter}$ & & Mmol/liter & $\mu \mathrm{mol} /$ liter & & $\mu \mathrm{mol} /$ liter & umol/liter & \\
\hline 1 & 1.40 & 0.40 & 3.5 & 1.25 & 0.62 & 2.0 & 1.50 & 0.85 & 1.8 \\
\hline 2 & 6.50 & 0.55 & 11.8 & 3.65 & 0.53 & 6.9 & 8.75 & 1.23 & 7.1 \\
\hline 3 & 2.25 & 0.12 & 18.8 & 2.90 & 0.78 & 3.7 & 2.75 & 1.10 & 2.5 \\
\hline 4 & 15.75 & 1.60 & 9.8 & 5.00 & 0.80 & 6.3 & 6.60 & 1.20 & 5.5 \\
\hline 5 & 4.75 & 0.40 & 11.9 & 2.25 & 0.48 & 4.7 & 4.90 & 1.55 & 3.2 \\
\hline Mean \pm SEM & $6.13 \pm 2.57$ & $0.61 \pm 0.26$ & $11.2 \pm 2.5$ & $3.01 \pm 0.63$ & $0.64 \pm 0.06$ & $4.7 \pm 0.9$ & $4.90 \pm 1.30$ & $1.19 \pm 0.11$ & $4.0 \pm 1.0$ \\
\hline
\end{tabular}

- $P$, portal venous concentration; $V$, peripheral venous concentration; $P / V$, ratio between portal venous and peripheral venous serum concentration. 


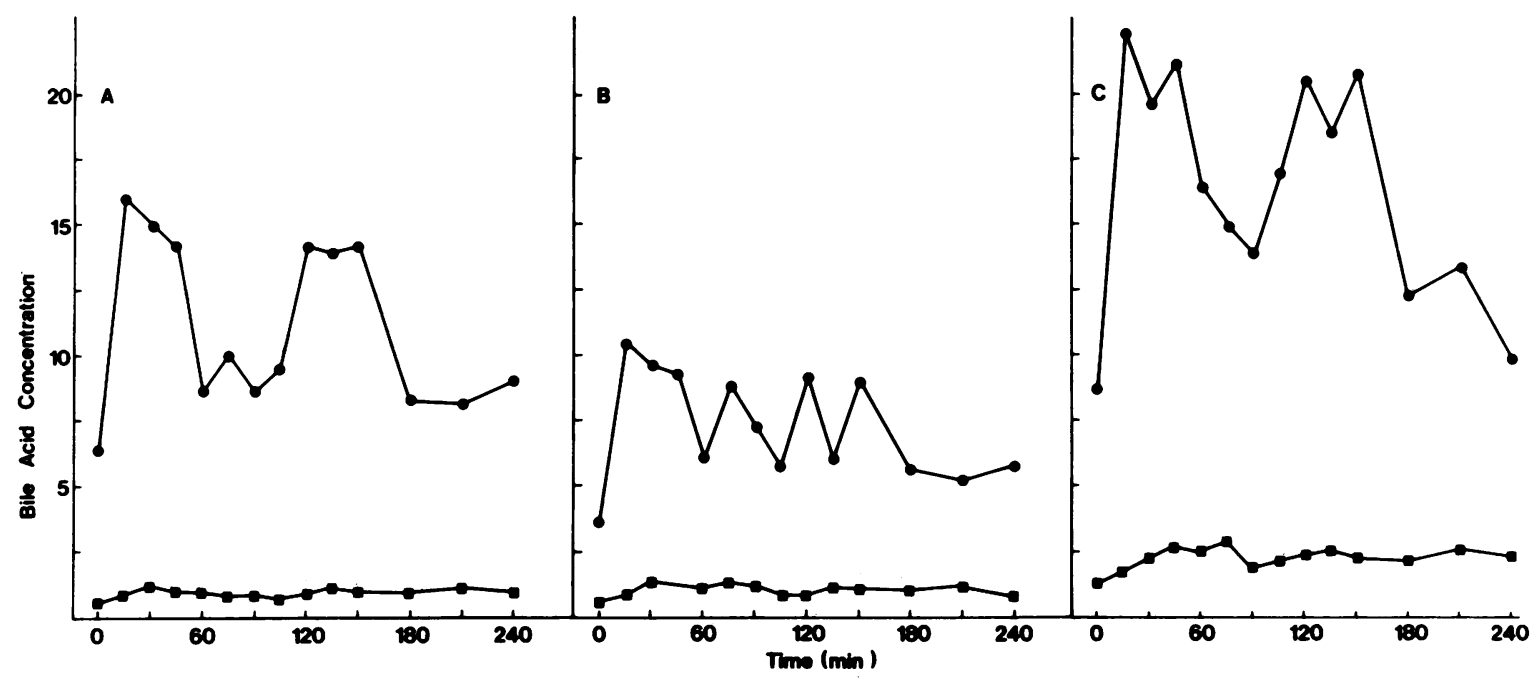

Figure 1 Concentrations of (A) cholic acid, (B) chenodeoxycholic acid, and (C) deoxycholic acid in portal venous $(O)$ and peripheral venous $(\square)$ serum in response to a standardized meal given at time 0 (patient 2). Bile acid concentration (micromoles/liter).

dividual bile acid reflected the simultaneous portal the relationship was somewhat different in this case. venous concentration (Fig. $3 \mathrm{~A}-\mathrm{C}$ ). As a consequence The relative composition of the individual bile acids of the lower uptake of the bile acids in patient no. 1, varied both between the different subjects and as a

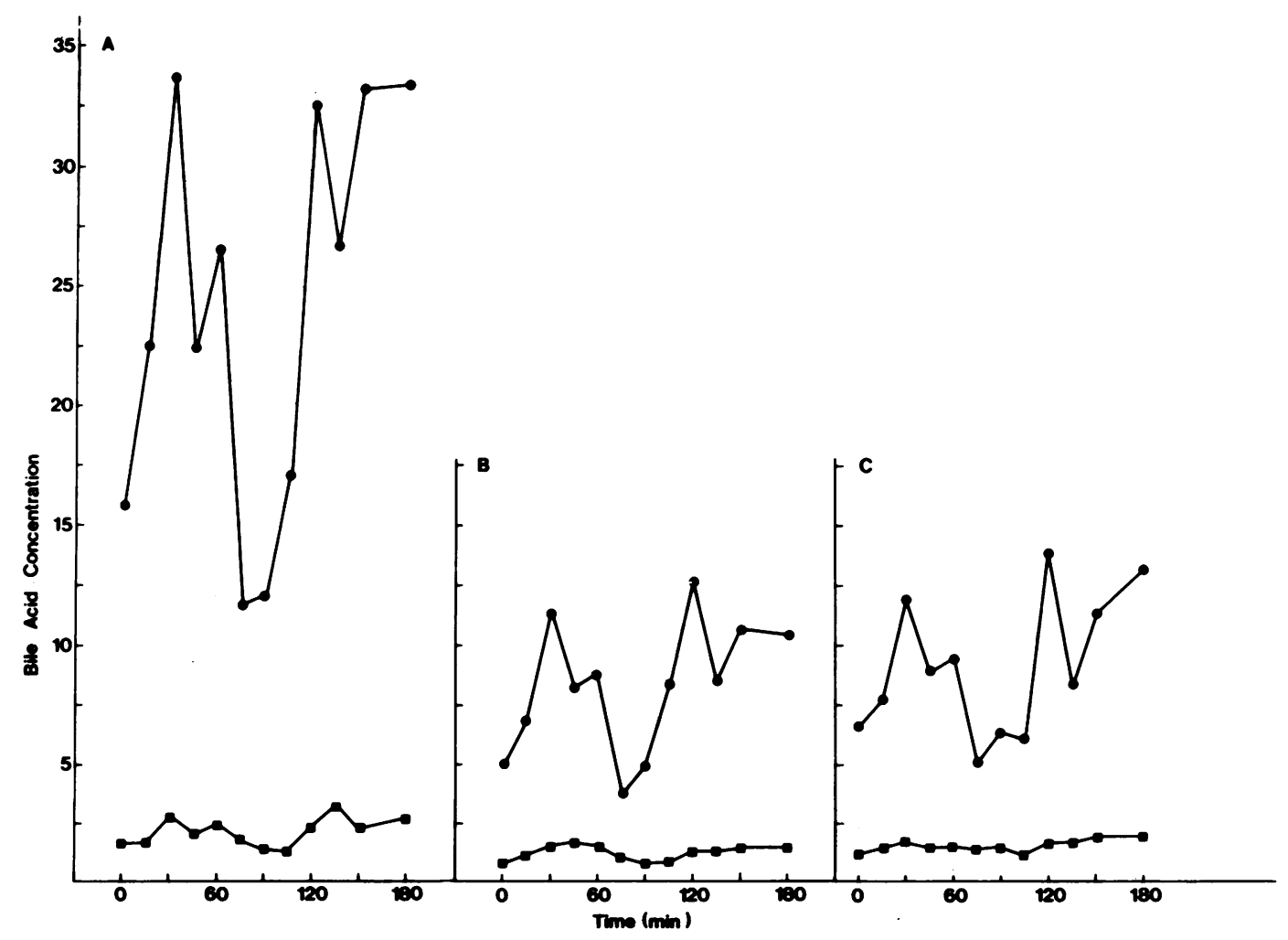

FIGURE 2 Concentrations of (A) cholic acid, (B) chenodeoxycholic acid, and (C) deoxycholic acid in portal venous $(\bullet)$ and peripheral venous $(\square)$ serum in response to a standardized meal given at time 0 (patient 4 ). Bile acid concentration (micromoles/liter). 
TABLE III

Maximal Postprandial Concentrations of Individual Bile Acids in Portal Venous and Peripheral Venous Serum of Five Cholecystectomized Subjects ${ }^{\circ}$

\begin{tabular}{|c|c|c|c|c|c|c|c|c|c|c|}
\hline \multirow[b]{2}{*}{ Patient no. } & \multirow[b]{2}{*}{ Time } & \multicolumn{3}{|c|}{ Cholic acid } & \multicolumn{3}{|c|}{ Chenodeoxycholic acid } & \multicolumn{3}{|c|}{ Deoxycholic acid } \\
\hline & & $\mathbf{P}$ & $\mathrm{v}$ & $P / V$ & $\mathbf{P}$ & $\mathrm{v}$ & $\mathrm{P} / \mathrm{V}$ & $\mathbf{P}$ & $\mathrm{v}$ & $\mathrm{P} / \mathrm{V}$ \\
\hline & $\min$ & $\mu \mathrm{mol} /$ liter & $\mu \mathrm{mol} /$ liter & & umol/liter & 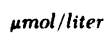 & & $\mu \mathrm{mol} /$ liter & $\mu \mathrm{mol} / \mathrm{liter}$ & \\
\hline 1 & 30 & 8.50 & 1.70 & 5.0 & 8.15 & 2.32 & 3.5 & 5.90 & 2.03 & 2.9 \\
\hline 2 & 15 & 16.00 & 0.90 & 21.1 & 10.40 & 0.80 & 13.0 & 22.25 & 1.70 & 13.1 \\
\hline 3 & 60 & 16.60 & 0.60 & 27.7 & 10.50 & 1.22 & 8.6 & 9.40 & 1.75 & 5.4 \\
\hline 4 & 30 & 33.85 & 2.78 & 12.2 & 11.50 & 1.55 & 7.4 & 12.00 & 1.70 & 7.1 \\
\hline 5 & 45 & 17.15 & 1.75 & 9.8 & 8.00 & 1.77 & 4.5 & 25.00 & 3.55 & 7.0 \\
\hline \multirow[t]{2}{*}{ Mean \pm SEM } & & 18.42 & 1.55 & 15.2 & 9.71 & 1.53 & 7.4 & 14.91 & 2.15 & 7.1 \\
\hline & & \pm 4.17 & \pm 0.38 & \pm 4.1 & \pm 0.70 & \pm 0.26 & \pm 1.7 & \pm 3.71 & \pm 0.36 & \pm 1.7 \\
\hline
\end{tabular}

- Time, time of maximal concentration (first peak) in relation to meal; $P$, portal venous concentration; $V$, peripheral venous concentration; $P / V$, ratio between portal venous and peripheral venous serum concentration.

consequence of the meal. Nevertheless, the sum of $\mathrm{C}$, $\mathrm{CD}$, and D ("total bile acids") in peripheral circulation correlated with the total concentration in the portal vein (Fig. 4).

\section{DISCUSSION}

The technique used for determination of the serum concentrations of $\mathrm{C}, \mathrm{CD}$, and $\mathrm{D}$ used in the present work is very similar to the one described previously by us $(5,7,10)$. The inclusion of one deuterium-labeled internal standard for each of the three bile acids, as well as the monitoring of specific ion pairs for each bile acid, would theoretically further improve the accuracy of the method (10). It should be pointed out

TABLE IV

Fasting Concentrations of Individual Bile Acids in Hepatic Venous and Peripheral Arterial Blood Serum of Five Healthy Subjects ${ }^{\circ}$

\begin{tabular}{|c|c|c|c|c|c|c|}
\hline \multirow{2}{*}{$\begin{array}{c}\text { Subject } \\
\text { no. }\end{array}$} & \multicolumn{2}{|c|}{ Cholic acid } & \multicolumn{2}{|c|}{$\begin{array}{l}\text { Chenodeoxycholic } \\
\text { acid }\end{array}$} & \multicolumn{2}{|c|}{ Deoxycholic acid } \\
\hline & HV & A & HV & A & HV & $\mathbf{A}$ \\
\hline & \multicolumn{2}{|c|}{$\mu \mathrm{mol} /$ liter } & \multicolumn{2}{|c|}{ 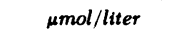 } & \multicolumn{2}{|c|}{ 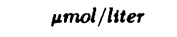 } \\
\hline 1 & 2.05 & 2.08 & 0.63 & 0.64 & 2.30 & 2.30 \\
\hline 2 & 0.39 & 0.42 & 1.13 & 1.34 & 0.94 & 1.17 \\
\hline 3 & 0.20 & 0.23 & 0.45 & 0.50 & 0.90 & 0.75 \\
\hline 4 & 0.38 & 0.35 & 2.25 & 2.55 & 0.70 & 0.62 \\
\hline 5 & 0.20 & 0.72 & 0.43 & 0.55 & 0.73 & 0.75 \\
\hline
\end{tabular}

- Hepatic venous (HV) and arterial (A) blood was withdrawn simultaneously during an investigation of carbohydrate metabolism in healthy volunteers (cf. 11). Bile acid concentrations were determined as described in Methods. that, under the present conditions, no information is achieved with respect to the degree and nature of conjugation or sulfation of the serum bile acids. This possible limitation of the methodology used is discussed below.

In the present work, fasting and postprandial levels of $C, C D$, and $D$ were determined in portal venous and peripheral venous circulation of patients with cholesterol gallstone disease 5-7 d after cholecystectomy. Even if the patients were fully recovered from the operation, it is reasonable to assume that the bile acid pool is acutely depleted by cholecystectomy, and that compensatory changes may not have taken place completely as soon as 1 wk after surgery. This probably explains why the fasting concentrations of the bile acids, particularly $\mathrm{CD}$, were somewhat lower than those previously reported $(5,7,10)$. In agreement with previous work $(6,12)$, our study of cholecystectomized subjects showed an earlier and less distinct peak in peripheral serum bile acid concentrations in response to a meal compared to what is seen in subjects with an intact gallbladder $(6,7,12)$. This is presumably due to the localization of the bile acid pool to the intestine, resulting in a more prompt response to feeding. It is reasonable to speculate that the second peak often seen represents absorption of resecreted bile acids.

This study demonstrated a considerable increase in portal venous bile acid concentration during a meal. The increment in bile acid inflow to the liver is actually still higher because the splanchnic circulation is also increased in response to feeding (13). If hepatic blood flow is presumed to be $1,000 \mathrm{ml} / \mathrm{min}$ in the fasting state, and $1,500 \mathrm{ml} / \mathrm{min}$ during a meal, the present values would correspond to a hepatic uptake (and secretion) of $\sim 300 \mu \mathrm{mol} / \mathrm{h}$ in fasting and maximum 
TABLE V

Estimated Hepatic Fractional Uptake of Individual Bile Acid Classes

in Five Cholecystectomized Patients ${ }^{\circ}$

\begin{tabular}{|c|c|c|c|c|c|c|}
\hline \multirow[b]{2}{*}{ Patient no. } & \multicolumn{2}{|c|}{ Cholic acid } & \multicolumn{2}{|c|}{ Chenodeoxycholic acid } & \multicolumn{2}{|c|}{ Deoxycholic acid } \\
\hline & Fasting & Postprandial & Fasting & Postprandial & Fasting & Postprandial \\
\hline & \multicolumn{6}{|c|}{$\%$} \\
\hline 1 & 65 & 75 & 43 & 65 & 36 & 59 \\
\hline 2 & 89 & 93 & 82 & 90 & 82 & 90 \\
\hline 3 & 93 & 95 & 67 & 85 & 53 & 77 \\
\hline 4 & 87 & 89 & 80 & 83 & 77 & 82 \\
\hline 5 & 89 & 87 & 73 & 73 & 62 & 82 \\
\hline Mean \pm SEM & $85 \pm 5$ & $88 \pm 4$ & $69 \pm 7$ & $79 \pm 4$ & $62 \pm 8$ & $78 \pm 5$ \\
\hline
\end{tabular}

- Calculated as $\frac{0.75 \times \mathrm{P}+0.25 \times \mathrm{V}-\mathrm{V}}{0.75 \times \mathrm{P}+0.25 \times \mathrm{V}} \times 100 \%$, where $\mathrm{P}$ is portal venous and $\mathrm{V}$ peripheral venous concentration. See reference 5 for details.

$1,400 \mu \mathrm{mol} / \mathrm{h}$ during a meal. The former figure is slightly lower than the calculated nocturnal bile acid secretion in healthy man, 350-900 $\mu \mathrm{mol} / \mathrm{h}$ (14-17). The latter figure is in reasonable agreement with the reported values for stimulated bile acid secretion in cholecystectomized man, $\sim 1,100 \mu \mathrm{mol} / \mathrm{h}$ (18).

In spite of increases in the portal venous bile acid concentration up to almost $60 \mu \mathrm{mol} /$ liter, we did not observe any signs of saturation of the hepatic uptake system. Thus, the portal venous/peripheral venous ra- tio and the estimated fractional uptake remained constant-and higher for $\mathrm{C}$ than for $\mathrm{CD}$ or $\mathrm{D}$-during maximal inflow of bile acids. It should be emphasized that, although the calculation of fractional uptake assumes a constant proportion between hepatic arterial and portal venous blood flow, the possible error that could be introduced by variation of this flow ratio is very limited. As mentioned above, unconjugated bile acids are not separated from conjugated with the methodology used in this work. Although the propor-

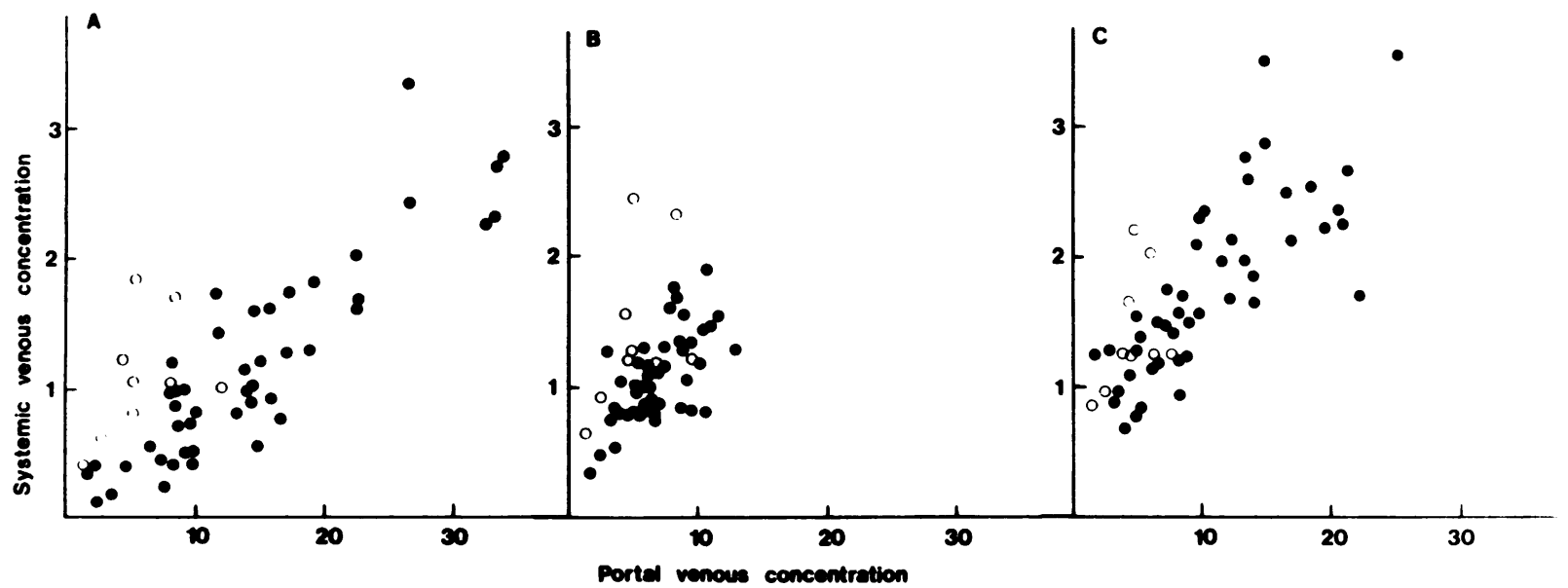

Figure 3 Relation between portal venous and peripheral venous serum concentrations of (A) cholic acid, (B) chenodeoxycholic acid, and (C) deoxycholic acid in five cholecystectomized patients. Samples were taken before and during a standardized meal. Patient 1 , who had a slightly elevated serum bilirubin level, is shown with open circles. The correlation coefficients $(r)$ were (values calculated excluding patient 1 within parentheses) for cholic acid $+0.80(+0.87)$, for chenodeoxycholic acid $+0.45(+0.62)$, and for deoxycholic acid $+0.75(+0.77)$. The correlations were in all cases significant at the $P<0.001$ level. Systemic and portal venous concentrations (micromoles/liter). 


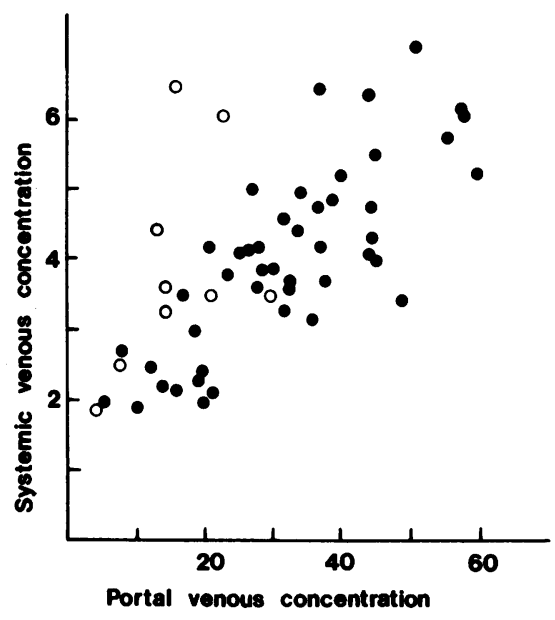

Figure 4 Relation between portal venous and peripheral venous serum concentrations of "total bile acids" (sum of cholic acid, chenodeoxycholic acid, and deoxycholic acid) in five cholecystectomized patients. Symbols as in Fig. 3. The correlation coefficient $(r)$ was +0.64 (excluding patient $1,+0.73)$, in both cases $P<0.001$. Systemic and portal venous concentrations (micromoles/liter).

tion of unconjugated bile acids in the portal vein is probably small (cf. 19), the fractional uptake of these bile acids is less than that of conjugated bile acids (20). Unconjugated bile acids are always returning to the liver from the intestine, and the fraction of portal bile acids in unconjugated form might be greater in cholecystectomized patients. It might also be greater during the fasting state, which may explain why, at least for $D$, the average fractional uptake values tended to be higher in the postprandial state. Thus, the fractional hepatic extraction estimates in Table $V$ represent the geometric means of the hepatic fractional extraction values for the individual species (unconjugated, glycine conjugate, taurine conjugate) of each of the bile acid classes. Even with this reservation, however, it is reasonable to assume that the difference in uptake between tri- and dihydroxy bile acid classes is related to a different affinity of the hepatic transport system for different bile acids, as has been demonstrated in the rat (21), and/or to differences in protein binding (22). The additional possibility of a difference in the lymphatic transport between trihydroxy and dihydroxy bile acid has been raised by Lindblad et al. (23). However, recent studies on lymphatic bile acid transport in man demonstrated that $<0.1 \%$ of bile acid enterohepatic circulation occurs via this route. ${ }^{2}$

Thus, the present results show that saturation of the hepatic bile acid uptake cannot be reached under

\footnotetext{
${ }^{2}$ Ewerth, S., I. Björkhem, K. Einarsson, and L. Öst. 1982 J. Lipid Res. In press.
}

physiologically occurring conditions in man. This concept is in agreement with data obtained from studies in the dog (24-26) and in the rat $(21,27)$. The estimated fractional uptake of bile acids was reduced in one of our patients (No. 1). It is of interest to note, that this subject did not display increased levels of peripheral bile acids in the fasting or in the postprandial state. This underscores the difficulty to identify subjects with a moderately reduced hepatic bile acid uptake by analysis of serum bile acids even after a load of endogenous bile acids (cf. 28).

A major new finding of the present work was that peripheral venous concentrations of individual bile acids reflect the simultaneous portal venous concentration. There was also a fair correlation between total bile acids at the two sampling sites. The latter finding supports and extends the reports by Lindblad et al. (23) and Paré et al. (29). Using less specific techniques, these authors found parallel increases in portal venous and peripheral venous concentrations of total bile acids in response to feeding or to cholecystokinin administration in patients with malignant disease or with alcoholic liver disease. The present data strongly support the concept that variation in the intestinal "input" of bile acids is the major determinant of peripheral serum bile acid concentrations in healthy man (5-7, 30). Furthermore, the portal venous inflow of bile acids can be monitored indirectly with some certainty by analysis of peripheral venous samples. This assumption has been made in several recent studies on the enterohepatic circulation of bile acids, but has never been directly validated in man previously (cf. 1).

In conclusion, our study confirms the presence of an efficient enterohepatic circulation of bile acids in man. The presence of a highly efficient hepatic uptake process, which has higher affinity for trihydroxy than for dihydroxy bile acids, and which cannot be saturated under physiological conditions, is demonstrated. Measurement of peripheral serum bile acid concentrations reflect portal venous levels, and can thus give important information on the status of the enterohepatic circulation.

\section{ACKNOWLEDGMENTS}

We thank Ms. Gunvor Alvelius for expert technical assistance, Dr. Anders Juhlin-Dannfelt and Dr. Leif Tokics for providing the hepatic venous blood samples, and Ms. Lena Ericsson for skillful preparation of the manuscript.

This study was supported by grants from the Swedish Medical Research Council (03X-04793), the Loo and Hans Osterman Foundation, the Swedish Society of Medical Sciences, and Karolinska Institutet.

\section{REFERENCES}

1. Hofmann, A. F. 1976. The enterohepatic circulation of bile acids in man. Adv. Intern. Med. 21: 501-534. 
2. Small, D. M. 1976. The etiology and pathogenesis of gallstones. Adv. Surg. 10: 63-85.

3. Danielsson, H., and J. Sjövall. 1975. Bile acid metabolism. Annu. Rev. Biochem. 44: 233-253.

4. Grundy, S. M. 1978. Cholesterol metabolism in man. West. J. Med. 128: 13-25.

5. Ahlberg, J., B. Angelin, I. Björkhem, and K. Einarsson. 1977. Individual bile acids in portal venous and systemic blood serum of fasting man. Gastroenterology. 73: 13771382.

6. La Russo, N. F., M. G. Korman, N. E. Hoffman, and A. F. Hofmann. 1974. Dynamics of the enterohepatic circulation of bile acids. Postprandial serum concentrations of conjugates of cholic acid in health, cholecystectomized patients, and patients with bile acid malabsorption. N. Engl. J. Med. 291: 689-692.

7. Angelin, B., and I. Björkhem. 1977. Postprandial serum bile acids in healthy man-evidence for differences in absorptive pattern between individual bile acids. Gut. 18: 606-609.

8. Ahlberg, J., B. Angelin, and K. Einarsson. 1981. Hepatic 3-hydroxy-3-methylglutaryl coenzyme A reductase activity and biliary lipid composition in man: relation to cholesterol gallstone disease and effects of cholic acid and chenodeoxycholic acid treatment. J. Lipid Res. 22: 410-422.

9. Ewerth, S., and B. Angelin. 1980. Cannulation of the umbilical vein in adult man. A review of surgical techniques, possible complications and clinical applications. Acta Chir. Scand. 500(Suppl.): 75-78.

10. Angelin, B., I. Björkhem, and K. Einarsson. 1978. Individual serum bile acid concentrations in normo- and hyperlipoproteinemia as determined by mass fragmentography: Relation to bile acid pool size. J. Lipid Res. 19: 527-537.

11. Felig, P., and J. Wahren. 1971. Influence of endogenous insulin secretion on splanchnic glucose and amino acid metabolism. J. Clin. Invest. 50: 1702-1711.

12. Schalm, S. W., N. F. La Russo, A. F. Hofmann, N. E. Hoffman, G. P. van Berge Henegouwen, and M. G. Korman. 1978. Diurnal serum levels of primary conjugated bile acids. Assessment by specific radioimmunoassays for conjugates of cholic and chenodeoxycholic acid. Gut. 19: 1006-1014

13. Richardson, P. D. I., and P. G. Withrington. 1981. Liver blood flow. I. Intrinsic and nervous control of liver blood flow. Gastroenterology. 81: 159-173.

14. Brunner, H., T. C. Northfield, A. F. Hofmann, V. L. W. Go, and W. H. J. Summerskill. 1974. Gastric emptying and secretion of bile acids, cholesterol, and pancreatic enzymes: duodenal perfusion studies in healthy subjects. Mayo Clinic Proc. 49: 851-860.

15. La Russo, N. F., N. E. Hoffman, A. F. Hofmann, T. C. Northfield, and J. L. Thistle. 1975. Effect of primary bile acid ingestion on bile acid metabolism and biliary lipid secretion in gallstone patients. Gastroenterology. 69: 1301-1314.

16. Northfield, T. C., and A. F. Hofmann. 1975. Biliary output during three meals and an overnight fast. I. Relationship to bile acid pool size and cholesterol saturation of bile in gallstone and control subjects. Gut. 16: 1-11.

17. Mok, H. Y. I., K. von Bergmann, and S. M. Grundy. 1980. Kinetics of the enterohepatic circulation during fasting: biliary lipid secretion and gallbladder storage. Gastroenterology. 78: 1023-1033.

18. Shaffer, E. A., and D. M. Small. 1977. Biliary lipid secretion in cholesterol gallstone disease. The effect of cholecystectomy and obesity. J. Clin. Invest. 59: 828-840.

19. Makino, I., S. Nakagawa, and K. Mashimo. 1969. Conjugated and unconjugated serum bile acid levels in patients with hepatobiliary diseases. Gastroenterology. 56: 1033-1039.

20. Gilmore, I. T., and R. P. H. Thompson. 1978. Direct measurement of the first pass extraction of bile acids by the liver in man Gut. 19: A971 (Abstr.).

21. Reichen, J., and G. Paumgartner. 1976. Uptake of bile acids by perfused rat liver. Am. J. Physiol. 231: 734742.

22. Rudman, D., and F. E. Kendall. 1957. Bile acid content of human serum. II. The binding of cholanic acids by human plasma proteins. J. Clin. Invest. 36: 538-542.

23. Lindblad, L., K. Lundholm, and T. Scherstén. 1977. Bile acid concentrations in systemic and portal serum in presumably normal man and in cholestatic and cirrhotic conditions. Scand. J. Gastroenterol. 12: 395-400.

24. O'Maille, E. R. L., T. G. Richards, and A. H. Short. 1967. The influence of conjugation of cholic acid on its uptake and secretion: hepatic extraction of taurocholate and cholate in the dog. J. Physiol. (Lond.) 189: 337-350.

25. Glasinovic, J. C., M. Dumont, M. Duval, and S. Erlinger. 1975. Hepatocellular uptake of taurocholate in the dog. J. Clin. Invest. 55: 419-426.

26. Pries, J. M., C. A. Sherman, G. C. Williams, and R. F. Hanson. 1979. Hepatic extraction of bile salts in conscious dogs. Am. J. Physiol. 236: E191-E197.

27. Reichen, J., and G. Paumgartner. 1975. Kinetics of taurocholate uptake by the perfused rat liver. Gastroenterology. 68: 132-136.

28. Gilmore, I. T., and A. F. Hofmann. 1980. Altered drug metabolism and elevated serum bile acids in liver disease: a unified pharmacokinetic explanation (Editorial). Gastroenterology. 78: 177-179.

29. Paré, P., J. C. Hoeffs, and M. Aschcavai. 1981. Determinants of serum bile acids in chronic liver disease. Gastroenterology. 81: 959-964.

30. La Russo, N. F., N. E. Hoffman, M. G. Korman, A. F. Hofmann, and A. E. Cowen. 1978. Determinants of fasting and postprandial serum bile acid levels in healthy man. Dig. Dis. Sci. 23: 385-391. 